Este libro forma parte del acervo de la Biblioteca Jurídica Virtual del Instituto de Investigaciones Jurídicas de la UNAM

\title{
HACIA UN COSMOPOLITISMO SANITARIO ANTE LA EMERGENGIA DEL COVID-19
}

\author{
Jorge Enrique LINARES SALGADO
}

\begin{abstract}
SUMARIO: I. Introducción. II. La crisis ecosocial y sanitaria después de la pandemia. III. El cosmopolitismo moderno. IV. La reforma de los organismos internacionales. V. Los principios de los sistemas públicos de atención a la salud. VI. La necesidad de un sistema de salud pública con acceso universal ante la pandemia del COVID-19 en México. VII. Referencias
\end{abstract}

\section{INTRODUCGIÓN}

En este artículo me propongo argumentar la necesidad de un cosmopolitismo ambientaly sanitario; es decir, un modelo de ética y política de acuerdos mínimos de alcance mundial, que sea capaz de estructurar un orden geopolítico emergente para encarar los enormes retos de la pandemia del COVID-19.

Desde sus inicios la bioética ha tenido una vocación internacionalista y, podemos decir, genuinamente cosmopolita como base de una ética civil planetaria. ${ }^{1}$ Ante la falta de una teoría ética unificada en nuestra época, la comunidad internacional había podido llegar a consensos y compromisos éticos de alcance global, mediante las propuestas de organismos como la Organización de las Naciones Unidas (ONU), la Organización de las Naciones Unidas para la Educación, la Ciencia y la Cultura (UNESCO), la Organización Mundial de la Salud (OMS) o la Asociación Médica Mundial. Tales consensos, surgidos de la voluntad de distintas posiciones políticas para lograr acuerdos mínimos, siguen siendo el paradigma de la de-

$1 \quad \mathrm{Al}$ surgimiento de la bioética antecedieron códigos éticos mundiales que comenzaron a regular la praxis y la investigación biomédicas: la Declaración Universal de los Derechos Humanos (1948), el Código de Nuremberg (1947), o el código que proclamó la Asociación Médica Mundial: la Declaración de Helsinki (1964). Esos códigos éticos conformaron los cimientos de nuevas normatividades de carácter convencional, pero de alcance verdaderamente global. 
Este libro forma parte del acervo de la Biblioteca Jurídica Virtual del Instituto de Investigaciones Jurídicas de la UNAM

liberación bioética, tanto en una pequeña escala (un comité de bioética de un hospital o un centro de investigación) como a gran escala (los comités de bioética nacionales o mundiales). La Declaración Universal sobre Bioética y Derechos Humanos (2005) de la UNESCO, a pesar de las reservas que puedan argumentarse y de que no es un documento vinculante, representa una última manifestación de la necesidad de bases y principios mundiales de carácter ético-político.

En la actualidad el poder de la tecnociencia no está al alcance de todos los pueblos ni beneficia a todas las personas, principalmente en cuanto a las tecnologías médicas y farmacéuticas. Por ello, es necesaria una reorientación social y política de ese poder médico y biotecnológico que, en muchas circunstancias, se distancia de las necesidades de los individuos y de las comunidades. El rumbo de la civilización tecnológica en la que vivimos lo dirigen unos cuantos que, en su ceguera y ambición desmedida, acaparan el poder económico y político, determinan las políticas públicas e impiden acuerdos internacionales para el desarrollo equitativo de todas las naciones.

No obstante, es innegable que el avance tecnocientífico de los últimos años ha contribuido a la integración de todas las naciones y pueblos en un solo mundo interrelacionado, con un único sistema económico global, y con graves y comunes problemas ecológicos y sociales. Pero dicha integración no se ha trasladado aún al plano ético y político. Por el contrario, ha provocado reacciones nacionalistas, etnocéntricas y tribalistas como hemos visto ante la emergencia sanitaria.

\section{LA CRISIS ECOSOCIAL Y SANITARIA DESPUÉS DE LA PANDEMIA}

La triple crisis global que estamos atravesando por la pandemia del COVID-19 no sólo es a) sanitaria (incluyendo la afectación a la salud mental), también es b) económica, y c) ética y política. Ha planteado un enorme desafío a la responsabilidad y a la cooperación internacional e intranacional. Sin embargo, la estrategia predominante para hacer frente a los problemas de orden global sigue siendo localista o, en el mejor de los casos, regional y se sujeta a los intereses de los Estadosnación, a la corta visión de los agentes mundiales de poder hegemónico. No predomina, pese a diversos esfuerzos surgidos en las últimas décadas, una razón práctica común (frónesis o prudencia práctica, como decía Aristóteles). Requerimos, por tanto, un ámbito cosmopolita para la ética y la política capaz de coordinar y organizar los esfuerzos para contender con las crisis ambientales y geopolíticas que tenemos 
Este libro forma parte del acervo de la Biblioteca Jurídica Virtual del Instituto de Investigaciones Jurídicas de la UNAM

que enfrentar, como el caso de una pandemia por un coronavirus nuevo, para el cual nadie poseía defensas naturales ni estaba preparado de manera efectiva.

Las crisis ecológicas globales (el cambio climático que se acelera, la pandemia del COVID-19 y otras posibles pandemias, la recesión económica, el crimen organizado y el narcotráfico con sus redes transnacionales, etc.) causan colapsos en las instituciones y normas ético-políticas, y tienen fundamento en el agotamiento de un modelo civilizatorio ligado a la acumulación y concentración del capital, es decir, a la privatización como tendencia contradictoria en un mundo interdependiente, más que al desarrollo equitativo de los seres humanos.

Una crisis sanitaria y social como la que se ha desatado hace indispensable construir el ámbito cosmopolita para poder resistir adecuada y oportunamente sus efectos negativos (recesión económica generalizada, saturación de sistemas públicos de salud, disputas por los medicamentos y vacunas, inestabilidad política, distorsiones y manipulación de la información pública, tensiones entre sociedad civil y gobiernos, controversias científicas y técnicas sobre tratamientos y vacunas efectivos, debates sobre los criterios éticos para la asignación de recursos médicos escasos, etc.). De otro modo, la política global se seguirá limitando a pactos y alianzas pasajeras de conveniencia que perpetúan a los mismos poderes hegemónicos, pero que nos embargan con una enorme deuda que deberán pagar muchos seres humanos, actuales y futuros, por los desastres ambiental, sanitario y socioeconómico extendidos.

Nos preguntamos cómo es posible una ética-política ambiental y sanitaria que sea cosmopolita; qué requisitos debe reunir y qué experiencias históricas permiten albergar la esperanza de que tenga efectividad. Este cosmopolitismo ambiental y sanitario debe fundarse en principios de justicia global e intergeneracional, así como en nuevas estructuras de democracia participativa mundial en varios niveles: local, regional y global. Por tanto, los principios que fundamentan la necesidad del cosmopolitismo en la situación actual de emergencia sanitaria son:

- mayor interdependencia y necesaria reciprocidad colectiva entre las naciones y personas;

- ampliación del alcance de nuestras responsabilidades individual y colectiva;

- precaución para detener y evitar daños a la biosfera, la salud pública y los sistemas tecnosociales; 
Este libro forma parte del acervo de la Biblioteca Jurídica Virtual del Instituto de Investigaciones Jurídicas de la UNAM

- protección de la biodiversidad, de la salud pública, la estabilidad política y económica, así como de la diversidad cultural y la pluralidad moral;

- participación ciudadana deliberativa, que implica la reinvención de las instituciones y mecanismos de las democracias representativas convencionales.

\section{EL COSMOPOLITISMO MODERNO}

El renacimiento del antiguo ideal cosmopolita aconteció, de hecho, durante la ilustración y se plasmó en la Declaración de los derechos del hombre y del ciudadano de la Revolución francesa de $1789 .{ }^{2}$ Unos cuantos años después emerge uno de los impulsos éticos más importantes al cosmopolitismo; se trata del ideal kantiano de una confederación de naciones republicanas que pacten un orden mundial hacia la Paz perpetua. Así, el cosmopolitismo moderno surgió, primordialmente, en la filosofia política de Immanuel Kant (1999) y ha continuado en las propuestas de autores contemporáneos que han seguido la misma orientación, como John Rawls (2005), Kwame Appiah (2007) o Thomas Pogge (2013). El propósito fundamental de un cosmopolitismo ambiental y sanitario es superar el "estado de naturaleza" en la geopolítica actual para construir una ciudadanía mundial y un estado de derecho cosmopolita ("Weltbürgerrecht") que proteja a todos por igual y que impulse, mediante el uso adecuado de la ciencia y la tecnología, el bienestar equitativo.

Kant define por primera vez el contenido normativo del cosmopolitismo moderno mediante el modelo de una confederación de Estados republicanos que se apeguen al derecho cosmopolita para preservar, cuanto sea posible, una paz duradera. Según Kant, este proyecto cosmopolita se basa en el hecho de que la Tierra es una esfera que nos vincula a todos en igualdad y que, por tanto, tenemos el mismo derecho a disfrutar de sus beneficios, así como a elegir el sitio de residencia que más convenga. Es el ideal de la hospitalidad y la igualdad universal de todas las personas, que sería la base de un derecho migratorio mundial. El imperativo categórico kantiano

2 Hay que señalar que en 1791 Olympe de Gouges formuló la Declaración universal de los derechos de la mujer y la ciudadana, como contraparte y complemento necesario de la Declaración original. Hoy más que nunca, las reivindicaciones de los movimientos feministas por la igualdad plena en términos jurídico-políticos y la equidad en términos sociales entre los dos sexos es un objetivo central del cosmopolitismo. Justamente las naciones y gobiernos que son más reactivos o contrarios a las políticas cosmopolitas son los que mantienen y preservan las costumbres, normas y políticas patriarcales de sometimiento y dominación de las mujeres en muchas naciones del orbe. 
Este libro forma parte del acervo de la Biblioteca Jurídica Virtual del Instituto de Investigaciones Jurídicas de la UNAM

reafirma el derecho cosmopolita a compartir la Tierra como patrimonio común y a que cada uno sea respetado como un fin en sí mismo en cualquier lugar del planeta.

Ahora bien, en la actualidad una confederación mundial de naciones tiene que implicar la participación ciudadana en la conducción y evaluación del desarrollo tecnocientífico y de sus aplicaciones sociales, económicas y ambientales. Conlleva, por ende, la transformación de las democracias representativas de las repúblicas actuales, de sus mecanismos internos de distribución del poder, de sus contrapesos, así como una revisión profunda de las bases de la legitimidad del poder público. Pero una democracia participativa cosmopolita no podría alcanzarse sólo desde una visión universalista abstracta o desde los intereses predominantes de los Estado-nación, pues la mayoría de los problemas que debe resolver son de carácter tanto global como local. Un cosmopolitismo renovado exige la construcción de un nuevo pacto mundial que se legitime directamente en la ciudadanía global. Es decir, la única manera de enfrentar las principales adversidades sociales y ambientales del mundo globalizado reside en una perspectiva cosmopolita, que implique acuerdos y normatividades generales aplicables internacionalmente mediante el convencimiento y la fuerza de todas las naciones conjuntas; pero, al mismo tiempo, parte desde un cosmopolitismo multicultural que proteja y revalore la pluralidad epistémica, cultural y moral de las comunidades humanas al interior de cada nación.

Ante este nuevo contexto mundial que ha traído la emergencia sanitaria es necesario repensar una vez más el añejo proyecto cosmopolita para conformar una comunidad mundial con un estado político global. Aquí únicamente me concentraré en argumentar sintéticamente su necesidad ineludible y señalar las limitaciones éticas que han impedido la realización de este ideal cosmopolita, pero esas mismas limitaciones constituyen, paradójicamente, la clave de su necesidad y su posibilidad.

Tiene sentido repensar una nueva forma de cosmopolitismo que no puede suplantar a los ethos convencionales de los pueblos, ni a las culturas ni a las identidades nacionales, pero que debe superponerse como una red envolvente capaz de desactivar la discordia y aminorar la creciente desigualdad entre naciones y comunidades. En la Antigüedad, el cosmopolitismo surgió como la afirmación de la autonomía individual frente a la comunidad política. Era un cosmopolitismo abstracto, escéptico o irónico, como el de los cínicos griegos. Ser un "ciudadano del cosmos" (cosmopolites) significaba renegar de la propia comunidad y de otras vecinas. Ahora tiene que reconstruirse como un modelo ético-político de efectividad práctica para edificar 
Este libro forma parte del acervo de la Biblioteca Jurídica Virtual del Instituto de Investigaciones Jurídicas de la UNAM

una nueva comunidad mundial, intercultural, diversa pero solidaria y justa. El cosmopolitismo contemporáneo resguarda también la dignidad y la autonomía inalienables de cada persona, pero no se opone a las culturas o a las etnias de origen, sino que relativiza sus valores y normas y, ante todo, critica su cerrazón e intolerancia. Pero el problema sigue siendo el mismo al que se enfrentaron los estoicos o los cínicos antiguos: ¿cómo ser un ciudadano del mundo si no existe un "estado político mundial"? Aún más, ¿es conveniente, es deseable que exista un tipo de "gobierno" mundial? Ya Kant sostenía que era no solo imposible sino indeseable que se erigiera un único gobierno mundial en la figura de un soberano o de un grupo de poder. La alternativa viable, como sostiene Kant en Hacia la paz perpetua, es la confederación de naciones "republicanas" (bien ordenadas jurídica y políticamente con una autoridad legítima) que puedan acordar políticas comunes y, ante todo, que pacten convenios de paz, cooperación y desarrollo para sus habitantes, a la vez que reconozcan el derecho cosmopolita de todos a recibir los beneficios básicos de los sistemas sociotécnicos, como son los servicios de salud y medios de atención a las enfermedades. El cosmopolitismo, en la tradición moderna, implica el derecho de comercio e intercambio pacíficos para buscar el bienestar (nunca la invasión de un territorio o la guerra comercial ni de conquista, como sucedió en el pasado) y el principio de hospitalidad global para todos los ciudadanos del planeta. La emergencia de una nueva enfermedad epidémica de alcance mundial hace resurgir la necesidad de una configuración cosmopolita de las relaciones internacionales y de los derechos básicos de toda la humanidad.

Según Kwame Anthony Appiah (2007), dos ideas básicas han alimentado a lo largo de la historia el cosmopolitismo: a) que tenemos obligaciones con todos los humanos, más allá del estrecho marco de nuestra comunidad cultural o política, b) que debemos considerar en igualdad los intereses de todas las personas, sin esperar ni desear que cada persona o cada sociedad deba converger en una sola forma de vida o vivir conforme a nuestros valores. El cosmopolitismo se basa en los ideales morales de una consideración universal y respeto a la diferencia entre las personas, no importando su nacionalidad o etnicidad, lengua, cultura o religión, sexo, preferencia sexual o identidad sexo-genérica.

Pero estos dos ideales suelen colisionar. O bien triunfa la diferencia como división y antagonismo que refuerza la intolerancia entre los grupos humanos, o bien se afirma una uniformidad $\mathrm{u}$ homogeneidad de modos de vida y valores con el fin de lograr la igualdad abstracta y la cohesión de una nación. El cosmopolitismo contemporáneo debe partir, según Ap- 
Este libro forma parte del acervo de la Biblioteca Jurídica Virtual del Instituto de Investigaciones Jurídicas de la UNAM

piah, de una idea simple: el hecho de que, en la comunidad fáctica de naciones, requerimos desarrollar hábitos de coexistencia pacífica: potenciar la conversación como forma de asociación, como forma de vida común entre las diferentes culturas y tradiciones. El cosmopolitismo responde así ante las posiciones extremas muy atractivas del etnocentrismo, nacionalismo, la xenofobia, y el chovinismo, que lamentablemente hemos visto "viralizarse" en la crisis sanitaria actual. Una vía básica para lograrlo es destacar valores comunes subyacentes a todas las culturas; valores y principios transculturales, intersubjetivos, para la convivencia social y ahora para la sostenibilidad mundial. Dichos valores y principios ${ }^{3}$ se encuentran expresados, de algún modo, en los principios de la bioética y en el sentido común que surge ante una situación de pandemia. Cualquier persona es consciente de que hoy deben prevalecer los valores de cooperación, responsabilidad, solidaridad y precaución sobre los valores de beneficio egoísta, lucro económico, nacionalismo, xenofobia, y sobre toda acción desesperada del "sálvese quien pueda".

Ahora bien, es cierto que, como muchos han criticado, se impuso durante el siglo XX una versión abstracta y parcial del cosmopolitismo moderno que fracasó en su vocación internacionalista. Esta forma tergiversada de cosmopolitismo fue en realidad un universalismo que trataba de imponer los valores e intereses de las naciones más poderosas de Occidente. Pero reveló su auténtica cara de particularismo colonial (es decir, se ha manifestado como occidentalismo) en muchas de sus acciones, convenciones y políticas de carácter mundial. Pero este fracaso no significa que la humanidad no se pueda poner de acuerdo de modo intercultural, con argumentos científicos y demostrables empíricamente, en aquellos principios y valores necesarios para construir una comunidad global ético-política. La catastrófica situación de crisis sanitaria y sus efectos nos obliga a ello hoy más que nunca.

Los rasgos negativos del universalismo occidental moderno, también denominado eurocentrismo colonialista, fueron:

a) Sistema restrictivo y excluyente, sólo entraban en dicho "contrato social" los varones propietarios, blancos, racionales, heterosexuales, y únicamente se formulaba para los seres humanos; quedaron excluidos las mujeres, los desposeídos (los que sólo poseen su propia fuerza

3 Los principios básicos de la bioética aplicables a la tecnomedicina e investigación biomédica: beneficencia, no maleficencia, justicia, autonomía. Podemos agregar los principios ambientales y tecnológicos de responsabilidad, precaución, justicia ambiental e intergeneracional, protección y remediación ambiental. Véase Linares 2008. 
Este libro forma parte del acervo de la Biblioteca Jurídica Virtual del Instituto de Investigaciones Jurídicas de la UNAM

de trabajo), los pueblos "salvajes" y todos los demás animales y seres vivos; pero también personas con discapacidades, las personas homosexuales y las personas transgénero y transexuales. Este modelo civilizatorio universalista se impuso por la violencia y la fuerza militar, la explotación económica y la hegemonía política.

b) El universalismo occidental se concibió a sí mismo como domesticador de la rebelde naturaleza animal del ser humano; el Estado era como un gran ogro civilizador, pero luego abandonó su proyecto de formación universal y homogénea. Con las políticas neoliberales también dejó atrás su papel rector en la educación, la ciencia, el desarrollo técnico o las instituciones de salubridad pública. Lo que habían logrado, principalmente, los Estados europeos de posguerra en la construcción de un estado de bienestar y de equidad social, se ha puesto en peligro con el avance del capitalismo depredador y monopólico en los últimos años. En particular, los sistemas públicos de salud han sufrido deterioros y obsolescencias graves, y han sido casi desmantelados, como ha sucedido en varios países de Latinoamérica.

c) Como consecuencia de que el universalismo occidental no propició el despliegue de una comunidad mundial equitativa y multicultural que revalorara y rescatara saberes y técnicas tradicionales efectivas, el desarrollo científico y tecnológico se sacralizó y se convirtió en un poder supremo que se subordinó a los intereses del capital y del dominio político. Por eso, la crisis de la pandemia se precipitó en una situación social en la que se extiende la desconfianza hacia las políticas de los gobiernos, las recomendaciones de la ciencia y las acciones sanitarias basadas en evidencias y estudios científicos. La crisis sanitaria nos tomó por sorpresa porque muchos pensaban que vivíamos aún en el mundo ordenado por las verdades científicas y las acciones racionales y justificadas de los gobiernos. Por el contrario, descubrimos que habitamos en el mundo de la manipulación mediática de la verdad, reducida ahora a "datos" sujetos a la interpretación, a la mera doxa sin suficiente demostración empírica, que cualquier puede creer o negar en las redes sociales, en un ambiente de virulentas reacciones sociales porque desconfiamos de un mundo común regido por una razón práctica colectiva. La crisis de ese universalismo epistémico representa el ocaso de la Ilustración y el eclipse de la razón pública autocrítica, plural y tolerante. ${ }^{4}$

4 Sin embargo, en los últimos 50 años emergieron muy diversos movimientos sociales, muchas disidencias locales que han cuestionado de raíz los fundamentos del dominio occi- 
Este libro forma parte del acervo de la Biblioteca Jurídica Virtual del Instituto de Investigaciones Jurídicas de la UNAM

Todo este contexto tiene sentido para comprender en qué terreno germinó la pandemia global de COVID-19, y con qué barreras socioculturales y políticas tendremos que lidiar. Ha llegado, pues, la hora de un nuevo proyecto cosmopolita que tiene como referencia la utopía de una comunidad política mundial. Pero esta vez ya no puede estar fundado en el dominio de unas naciones sobre otras, el saqueo colonial, la desigualdad en el acceso al conocimiento y a los beneficios de la ciencia y la tecnología, la explotación de la naturaleza y el exterminio de la biodiversidad; sino en unas bases éticopolíticas de autocontención de los impulsos "racionalizados" de barbarie, y en el control social y democrático del poder tecnocientífico y político en un debate público abierto y permanente.

En esta época del libre mercado global, de los monopolios trasnacionales y del comercio injusto, los poderes hegemónicos (el G8, los organismos financieros internacionales, el crimen organizado, los países con arsenales nucleares, los productores de petróleo, los acaparadores de medicamentos y vacunas, etc.) "gobiernan" y se imponen, pero no tienen ni la legitimidad ni la fuerza suficiente para controlar la dinámica imparable de la globalización en todas sus manifestaciones. Ha pasado a la historia la época de los grandes imperios que dominaron el planeta. No existe, pues, un estado de derecho mundial ni un "Estado político" internacional. En el plano global, pese a avances significativos, seguimos en un "estado de naturaleza": priva la ley del más fuerte. Esto será terrible si para el desarrollo y aplicación de las vacunas para la COVID-19 prevalece este sistema mundial de la desigualdad y la confrontación de intereses nacionales y regionales.

Podemos observar la paradoja de la situación actual. Por un lado, la colaboración científica entre diversos grupos de investigación, públicos y privados, y diferentes gobiernos ha posibilitado el desarrollo de vacunas en tiempo récord; por otro, subsiste la disputa y las tendencias egoístas de acaparar las vacunas para los países más ricos y los gobiernos más poderosos en beneficio solo de un sector de sus ciudadanos. Por tanto, no existe en este momento una autoridad, liderazgo ni un consenso mundial capaz de dirimir y resolver eficazmente, tanto los conflictos entre las naciones como los graves problemas ambientales, sanitarios y socioeconómicos. Pero la necesidad es creciente y crucial para asegurar el futuro de toda la humanidad.

dental del mundo y sus narrativas hegemónicas; así se iniciaron los movimientos feministas, ecologistas, el de los derechos civiles de minorías étnicas, de diversidad sexual, de comunidades indígenas marginadas, etc. La modernidad occidental se volvió multicultural, multimoral, plurisexual, multívoca y ello ha constituido la base de la crítica al modelo civilizatorio y a las acciones y políticas de los Estados. 
Este libro forma parte del acervo de la Biblioteca Jurídica Virtual del Instituto de Investigaciones Jurídicas de la UNAM

Nuestra comunidad planetaria aún carece de un Estado mundial o de una estructura política internacional adecuada. Todos los problemas de orden global, como ha sido claramente la pandemia del COVID-19, han desbordado por completo las capacidades y recursos de los Estados-nación (como ha sido evidente en las crisis y colapsamientos de los sistemas nacionales de salud, incluso de países poderosos como los EE. UU.). No existe en este momento una autoridad mundial capaz de dirimir y resolver eficazmente (no lo ha sido, a pesar de sus esfuerzos, la OMS ni la ONU en general), tanto los conflictos entre las naciones como los graves problemas socioeconómicos y sanitarios que se derivan de la pandemia.

Los principios que fundamentan la necesidad de una "macroética" cosmopolita son los de la interdependencia y la ampliación del alcance de nuestra responsabilidad individual y colectiva. Gracias a la red global de interacciones tecnológicas, cada persona puede afectar y ser afectada por cualquier otra en el mundo entero, como ha sucedido con la expansión planetaria del nuevo coronavirus en menos de tres meses; pero, además, la humanidad está dañando las condiciones de vida de muchas otras especies y de ecosistemas enteros, destruyéndolos y entrando en contacto peligroso con microrganismos y virus que estaban lejos del alcance de los seres humanos. Por ello, este nuevo cosmopolitismo tendrá que dejar de ser antropocéntrico; debemos hacernos cargo de la nueva responsabilidad por preservar las condiciones de supervivencia y equilibrio de los ecosistemas. Así, el cosmopolitismo sanitario supone cuatro proyectos y objetivos mundiales indispensables:

a) La construcción de un sistema mundial de atención a la salud, público y con costos razonables, con servicios gratuitos en situaciones de emergencia sanitaria, como el de una pandemia. Cada ciudadano del mundo tendría derecho a estar registrado en un sistema público de salud de su nación y a recibir tratamientos en cualquier parte del mundo, mediante un esquema de intercambios y copagos internacionales. El sistema de salud universal debe garantizar por lo menos la atención de los padecimientos de mayor alcance y afectación en cada nación, y la atención en situación de epidemias y pandemias. La obligación de cada ciudadano es contribuir, mediante el esquema tributario de su nación, con un impuesto adecuado a sus ingresos, participar en campañas de vacunación y mantenerse informado y concernido de las recomendaciones e indicaciones para el autocuidado de la salud. Ciertamente, las enfermedades derivadas de adicciones y de accidentes físicos por riesgos asumidos voluntariamente implicarán impuestos adicionales o contribuciones directas de los ciudadanos. 
Este libro forma parte del acervo de la Biblioteca Jurídica Virtual del Instituto de Investigaciones Jurídicas de la UNAM

b) Una autoridad internacional con capacidad de decisión global para establecer medidas necesarias de mitigación ante pandemias y situaciones catastróficas que repercutan en la salud pública.

c) Una comunidad unificada de investigación científica y tecnológica que no esté dominada por los intereses económicos de la industria médica y farmacéutica, mediante consorcios públicos internacionales, como el que se creó para la secuenciación del genoma humano.

d) Acuerdos de política económica mundial para regular las patentes de medicamentos y tecnologías médicas, y para suspenderlas en situación de emergencia sanitaria. Asimismo, acuerdos internacionales de orden jurídico para evitar la privatización vía patentes de organismos vivos no modificados genéticamente, genomas o partes del genoma humano o de otros animales y el intercambio de biotecnologías para el desarrollo humano, la agricultura, alimentación y salud, mediante patentes de uso común que eviten la privatización, la concentración y el lucro excesivo.

\section{LA REFORMA DE LOS ORGANISMOS INTERNACIONALES}

Los objetivos cosmopolitas de las Naciones Unidas quedaron establecidos desde su fundación en 1945 y siguen siendo plenamente vigentes. ${ }^{5}$ Estos objetivos deben ratificarse y ampliarse ahora con la clara conciencia de la responsabilidad humana por la salud pública mundial, la alimentación suficiente y adecuada, la preservación de los ecosistemas y la biodiversidad de la Tierra. A partir de la creación de la ONU y de sus múltiples instancias de mediación y negociación internacional, así como el surgimiento de organismos globales que comenzaron a ejercer poder sobre los Estados-nación (el FMI, el Banco Mundial, la OMC) y a contrarrestar poco a poco su pretendida soberanía absoluta, se ha ido formando una estructura, todavía incipiente, para contener los efectos negativos (algunos no intencionales) de la crisis ambiental y climática, y del conflicto político entre naciones. De este modo, a partir de la ONU se habían edificado los cimientos de una confederación mundial de naciones capaz de autorregularse mediante un auténtico "parlamento de la humanidad". Sin embargo, estos impulsos éticos hacia el cosmopolitismo no han tenido el éxito deseado y, en gran parte, se puede decir que entraron en retroceso al perderse el equilibrio

5 A saber: el mantenimiento de la paz y la seguridad internacionales, el fomento del progreso económico y social mediante la cooperación internacional, la promoción y defensa de los derechos humanos y el respeto a la libre determinación de los pueblos. 
Este libro forma parte del acervo de la Biblioteca Jurídica Virtual del Instituto de Investigaciones Jurídicas de la UNAM

geopolítico de la Guerra Fría. Las Naciones Unidas no cuentan con el poder autónomo ni con la capacidad operativa para ello (tampoco la Unión Europea). Cualquier acción global depende (y seguirá dependiendo) de la voluntad de las potencias mundiales y de su frágil capacidad de cooperación (EE. UU., China, Rusia, la UE o países emergentes que quisieron entrar en el juego geopolítico como Brasil, India o las potencias asiáticas). Por ello es requisito insuperable la transformación democrática de la $\mathrm{ONU}^{6}$ y de los organismos supranacionales como la OMS para conferirles mayor poder de acción e intervención, y para establecer nuevos mecanismos legítimos de equilibrios y contrapesos entre los países miembros, que redunden en una mayor equidad internacional.

La ONU, y sus instancias de decisión como el Consejo de Seguridad, ameritan ya una remodelación profunda para convertirla en un sistema parlamentario mundial en el que se expresen, deliberen y lleguen a acuerdos diversas tendencias políticas mundiales, como de hecho sucede en varios organismos internacionales. El derecho cosmopolita ha comprendido tres campos esenciales: la protección de los derechos humanos, la mediación para evitar o resolver las guerras (que incluye el juicio y sanción a los crímenes de guerra), y la sanción por los crímenes de lesa humanidad. ${ }^{7}$ Un cuarto y quinto campos son indispensables: 1) la conservación ambiental y la protección de la biodiversidad y del patrimonio biocultural que pertenece a toda la humanidad, y no a los Estados nacionales. 2) el acceso universal a los servicios de salud y la acción conjunta de políticas sanitarias ante situaciones de emergencia mundial, como la pandemia del COVID-19.

\section{LOS PRINCIPIOS DE LOS SISTEMAS PÚBLICOS DE ATENCIÓN A LA SALUD}

Parte esencial de un orden cosmopolita puede consistir en un sistema mundial de atención a la salud de acceso verdaderamente universal. Según la OMS, un sistema público de salud debería basarse en estos principios: ${ }^{8}$

\footnotetext{
6 No obstante, para que ese nuevo orden político global surja se requerirá de la voluntad de las potencias tradicionales (EE. UU., Gran Bretaña, Francia, China y Rusia), así como de las potencias emergentes (Alemania, Italia, Japón, Australia, India, Sudáfrica o Brasil y México) para respaldar los necesarios acuerdos mundiales de cooperación y desarrollo equitativo.

7 Existen algunas bases ético-jurídicas para la consolidación del derecho cosmopolita: la Declaración Universal de los Derechos Humanos, la Convención para la Prevención y Sanción del Delito de Genocidio, el Estatuto de la Corte Penal Internacional.

8 OMS, "Sistemas de salud", disponible en: https://wrerw.who.int/healthsystems/topics/es/.
} 
Este libro forma parte del acervo de la Biblioteca Jurídica Virtual del Instituto de Investigaciones Jurídicas de la UNAM

- Acceso universal y equitativo a los servicios de salud (prevención, promoción, tratamiento y rehabilitación), independientemente de la capacidad de pago individual;

- Justicia distributiva e igualdad de oportunidades en el acceso a tecnologías médicas, tratamientos, vacunas y medicamentos;

- Sustentabilidad financiera y subsidiariedad social (que podría ser mediante un impuesto mundial a todas las personas de ingreso medio a alto);

- Atención de las enfermedades de mayor incidencia, morbilidad y mortalidad, como las epidemias y pandemias;

- Atención especializada y prioritaria ante casos de urgencia o emergencia sanitaria;

- Reducción y prevención de riesgos y daños mediante políticas preventivas, educativas y de autocuidado de la salud;

- Personal sanitario suficiente y bien capacitado, en evaluación constante y con incentivos adecuados para mejorar su servicio;

- Investigación sobre las condiciones y problemas principales de la salud de cada comunidad;

- Desarrollo de sistemas de información médica personalizada para fomentar el cuidado responsable y la investigación epidemiológica;

- Protección de la autonomía de las personas, así como de su información privada en el uso de big data de carácter sanitario para monitorear enfermedades y detectar y controlar epidemias.

La OMS informa que cerca de la mitad de la población mundial carece de acceso integral a los servicios sanitarios básicos: "En algunos países, hasta el $11 \%$ de la población sufre este tipo de dificultad financiera grave cada año y hasta el 5\% se ve arrastrada a la pobreza. A nivel mundial, alrededor de 150 millones de personas sufren catástrofes financieras anualmente y 100 millones se ven obligadas a vivir por debajo del umbral de pobreza. Únicamente el 5-10\% de las personas están cubiertas en el África subsahariana y en Asia meridional, mientras que en los países de ingresos medios los índices de cobertura varían entre el 20\% y el 60\%". ${ }^{9}$ En 2005 todos los miembros de las Naciones Unidas habían acordado tratar de lograr la cobertura sanitaria universal a más tardar en 2030, en el marco de los Objetivos de Desarrollo Sostenible, pero esa meta aún está muy lejana. En 2010, un total de 79 países destinaron a la asistencia sanitaria menos del

9 OMS, "Cobertura sanitaria universal", disponible en: https://wrew.who.int/es/newesroom/fact-sheets/detail/universal-health-coverage-(uhc). 
Este libro forma parte del acervo de la Biblioteca Jurídica Virtual del Instituto de Investigaciones Jurídicas de la UNAM

10\% del gasto gubernamental. Sin embargo, tan solo 8 de los 49 países más pobres del mundo tendrán alguna posibilidad de financiar servicios básicos de salud ${ }^{10}$ con sus propios recursos. De entre las 30 naciones más pobladas del mundo, ${ }^{11}$ sólo 9 tienen un sistema de salud suficiente y podrían enfrentar adecuadamente una emergencia sanitaria. ${ }^{12}$

El objetivo de la cobertura sanitaria universal es asegurar que todas las personas reciban los servicios sanitarios que necesitan, sin tener que pasar penurias financieras. Para ello se requiere que el sistema de salud cosmopolita se desarrolle paulatinamente, de nación en nación, mediante convenios y acuerdos de cooperación y transferencia de tecnologías y de personal especializado. Las dificultades principales que señalan la OMS para la cobertura universal son: a) la baja disponibilidad de recursos, pues "ningún país, con independencia de su riqueza, ha sido capaz de garantizar a todas las personas el acceso inmediato a todas las tecnologías o intervenciones que puedan mejorar la salud o prolongar la vida"; b) la dependencia excesiva de los pagos directos en el momento en que la gente necesita asistencia, "aun disponiendo de algún tipo de seguro médico, puede ser obligatorio contribuir en forma de copagos, coaseguros o deducibles"; c) el uso ineficiente y no equitativo de los recursos, pues "se malgasta el 20-40\% de los recursos destinados a la salud". Así, la OMS planteó que los países deben recaudar los fondos suficientes, disminuir la dependencia de los pagos directos para financiar los servicios de salud y mejorar la eficiencia y la equidad, para evitar los desperdicios y disparidades. ${ }^{13}$

La realidad que reveló la pandemia es que en países en desarrollo los sistemas de salud están en malas condiciones, y por lo que no garantizan un

10 La OMS utiliza 16 servicios de salud esenciales como indicadores del nivel y la equidad de la cobertura en los países, que agrupa en cuatro categorías: salud reproductiva, de la madre, el recién nacido y los niños; enfermedades infecciosas; enfermedades no transmisibles; acceso a servicios hospitalarios básicos. OMS, op. cit.

11 Que suman cerca de 5,950 millones de habitantes, más del 77\% de la población mundial.

12 China, EE. UU. (parcialmente), Japón, Alemania, Reino Unido, Francia, Italia, Corea del Sur y España, y en parte Rusia. En conjunto, representan unos 2,381 millones de habitantes, solo el $30 \%$ de la población mundial. Países muy poblados (con más de 50 millones) que no tienen capacidades suficientes en sus sistemas de salud: India, Indonesia, Pakistán, Nigeria, Bangladesh, Brasil, México, Filipinas, Egipto, Etiopía, Vietnam, Irán, Turquía, República del Congo, Tailandia, Sudáfrica, Tanzania, Myanmar, Colombia, suman más del 46\% del total mundial. Estos datos de 2019 son de la ONU: World Population Prospects, disponible en: https://population.un.org/wpp/Download/Standard/Population/.

13 Objetivos primarios de la OMS para la cobertura universal: aprovechar al máximo las tecnologías y los servicios sanitarios, incrementar y pagar mejor al personal sanitario, mejorar la eficiencia hospitalaria; reduciendo los errores médicos; eliminar el desperdicio de recursos y la corrupción. 
Este libro forma parte del acervo de la Biblioteca Jurídica Virtual del Instituto de Investigaciones Jurídicas de la UNAM

acceso justo y de calidad, intensifican la desigualdad social y la injusticia, y afectan severamente los niveles de desarrollo humano y bienestar general de la población. Pero en algunos países desarrollados, como los EE.UU., una parte considerable de la población carece de acceso a la atención pública sanitaria por el predominio de la inequidad inherente al mercado, implicando una erogación personal muy alta para los sectores desprotegidos. Por ello, una condición y objetivo esencial de un orden cosmopolita es la cobertura de salud universal en un sistema global, en condiciones de equidad, costos justos, oportunidades y ayudas diferenciadas en función de las necesidades de cada sociedad y de cada persona. Ello implica la necesidad de establecer mecanismos de colaboración y cooperación médica ${ }^{14}$ internacional entre los sistemas públicos de salud, la producción de tecnologías médicas a costo accesible y el mejoramiento de las condiciones ambientales de vida, así como la investigación biomédica destinada a solucionar los más graves y extendidos problemas de salud de cada comunidad mediante consorcios público/privados, por ejemplo, para producir vacunas ante una nueva pandemia.

Los países que no puedan financiar sus propios sistemas públicos de salud deberían recibir ayudas directas de la comunidad internacional, como asistencia de personal sanitario en misiones y campañas especiales; no mediante créditos, sino una contribución vía un impuesto internacional a todas las personas con ingreso medio-alto y alto en el mundo entero. Asimismo, un sistema de salud global puede construirse progresivamente mediante financiamiento social, vía mayor recaudación de impuestos y aportaciones de las empresas, los gobiernos y las personas. No es posible que de un día para otro logremos ese sistema mundial de salud, pero sí es factible que comencemos a edificarlo con objetivos y principios básicos cosmopolitas. ${ }^{15}$

\section{LA NEGESIDAD DE UN SISTEMA DE SALUD PÚBLICA CON ACGESO UNIVERSAL ANTE LA PANDEMIA DEL COVID-19 EN MÉXICO}

La pandemia de COVID-19 ha puesto en dificultades extremas a los sistemas de salud por la saturación de sus UCI y la falta de atención a otras enferme-

14 Las misiones heroicas que realizan ONGs como Médicos sin fronteras (https://wrere. $m s f . m x /$, deberían constituir la norma de un sistema mundial de cooperación e intercambio de personal sanitario en los países más pobres, y no basarse en programas ideológicos y propagandísticos de algunas naciones.

15 Véase OMS, Informe sobre la salud en el mundo. La financiación de los sistemas de salud: El camino hacia la cobertura universal, 2010, pp. 8 y 9, disponible en: https://wwre.who.int/whr/2010/es/. 
Este libro forma parte del acervo de la Biblioteca Jurídica Virtual del Instituto de Investigaciones Jurídicas de la UNAM

dades graves. Por ello, se ha reavivado en muchos países el debate sobre la necesidad de conformar un sistema público de salud con acceso universal, equitativo y sustentable para contender con todo tipo de epidemias y, ante todo, emergencias sanitarias.

Los países con sistemas públicos de salud robustos y de mayor cobertura tienen la capacidad de superar eficazmente las emergencias sanitarias. A ello se suman el uso adecuado de tecnologías digitales para la vigilancia epidemiológica, sin que esto signifique el abuso por parte de los gobiernos para usarlos como medios de intrusión en la vida privada de las personas.

Ahora bien, en México la pandemia de COVID-19 puso de relieve un factor adicional de riesgo, pues también son epidémicas la diabetes, la obesidad y las enfermedades cardiovasculares. Era previsible que las personas con menos recursos que las padecen constituyan el grupo de mayor riesgo (además de los adultos mayores). El sistema de salud pública se ha venido transformando por efecto de políticas neoliberales que beneficiaron la mercantilización de la atención para sujetarlos al mercado global de fármacos y tecnologías médicas, en lugar de fortalecer la medicina social preventiva y el autocuidado, que son indispensables para mitigar el efecto de las epidemias. La política neoliberal favoreció al sistema privado y ahogó al sistema público por falta de inversión y de formación de personal médico competente y suficiente. Con ello se ha reafirmado el "estado natural" de injusticia que se mantiene en la mayoría de los países en desarrollo, en cuanto a la igualdad de oportunidades. Esta "selección natural" de las enfermedades incrementa las brechas de desigualdad y distribuyen inequitativamente sus efectos deletéreos.

Hoy es posible, sin embargo, con los recursos materiales y humanos de los que dispone México, que se amplíe la cobertura hasta que sea universal, de acuerdo con las necesidades específicas, y con las aportaciones económicas de los ciudadanos en la medida de sus posibilidades, sin que ello implique costos onerosos para las familias. Asimismo, es esencial incrementar la formación y justa distribución nacional de personal sanitario especializado. Pero la pregunta difícil de responder es ¿cómo financiar y sostener los sistemas de salud públicos de los países de desarrollo bajo, principalmente el África, sur de Asia y Latinoamérica? ¿Cómo formar más personal médico y de investigación biomédica que atienda las necesidades más urgentes?

México se encuentra, de acuerdo con la OMS, entre los 79 países que destinan a la asistencia sanitaria menos del 10\% del gasto presupuestal, y está en los últimos lugares entre los miembros de la Organización para la Coope- 
Este libro forma parte del acervo de la Biblioteca Jurídica Virtual del Instituto de Investigaciones Jurídicas de la UNAM

ración y el Desarrollo Económico (OCDE). ${ }^{16}$ Parte fundamental de la inversión debería destinarse no sólo a la infraestructura, sino también a la formación continua de personal médico. Todos los países han estado innovando en los últimos años formas mixtas de obtener ingresos para financiar sus sistemas salud, mediante mecanismos de recaudación de impuestos y de combinación de infraestructuras públicas y privadas. ${ }^{17}$ Tanto políticas intranacionales como convenios internacionales de intercambio de médicos podrían impulsar el desarrollo y combinación de servicios de salud básicos para enfermedades epidémicas y de mayor persistencia y gravedad. Podemos transitar del sistema de patentes y mercado tecnomédico mundial (caso de los ventiladores mecánicos) a un sistema de cooperación y de inversión públicaprivada para proveer los servicios de salud, junto con otros servicios básicos como energía, alimentos y agua potable.

Se debe buscar distribuir equitativamente el uso de los recursos sanitarios, siempre inferiores a la demanda y localizar los recursos en donde más se necesiten. Por ejemplo, los institutos nacionales de salud sólo se ubican en la Ciudad de México, cuando debería haber cinco regiones nacionales con sedes de estos hospitales de tercer nivel e investigación. A pesar de que México ha intentado la "cobertura universal" en lo formal, mediante la creación del Seguro Popular y ahora con el Instituto de Salud para el Bienestar (INSABI), que se suma a los sistemas IMSS, ISSSTE, ISSFAM, Marina, Pemex y los sistemas estatales, la cobertura es muy desigual y la capacidad de acceso a atención médica de calidad sigue siendo muy deficiente. Lamentablemente, podemos decir que en México sobrevivimos en el peor de los dos mundos posibles: por un lado, un sistema público con muchas carencias y deficiencias y, por otro, un sistema privado de calidad muy desigual, cuyos buenos servicios son absurdamente caros, los baratos de muy mala calidad, y en el que dominan las compañías privadas de seguros.

Para ello se requiere formar un consenso nacional para que por fin la salud pública sea una prioridad del Estado mexicano. De este modo, estaríamos en la ruta de contribuir a un sistema cosmopolita de salud pública, y en posibilidad de cooperar y de realizar intercambios con países menos

16 Según datos de la OCDE, México destina sólo el 5.9\% del Producto Interno Bruto (PIB) al sector salud, casi la mitad de lo que invierten países como Austria, Bélgica, Alemania, Suiza y casi una tercera parte de lo que gasta Estados Unidos.

17 También se ha considerado aplicar impuestos a la venta de tabaco y bebidas alcohólicas, así como de los alimentos procesados con altos contenidos de azúcares, grasas y conservadores que representan altos riesgos para la salud. Pero en México no han sido aplicados eficazmente. Hay países que han gravado incluso los servicios de telefonía celular, boletos de avión o transacciones financieras para este fin. 
Este libro forma parte del acervo de la Biblioteca Jurídica Virtual del Instituto de Investigaciones Jurídicas de la UNAM

desarrollados. En conclusión, la salud es tan prioritaria que no debería seguir dependiendo de los intereses del mercado global de medicamentos y tecnologías médicas, y de la extendida privatización y mercantilización de los servicios de salud. El desafío global consiste en fortalecer los sistemas públicos de salud en todo el mundo para volverlos más eficaces, equitativos, sustentables y accesibles para todos para avanzar hacia un cosmopolitismo sanitario.

\section{REFERENCIAS}

APPIAH, Kwame Anthony, 2007, Cosmopolitismo. La ética en un mundo de extraños, Buenos Aires, Katz.

Granja, Dulce María y Leyva, Gustavo, 2009, Cosmopolitismo. Democracia en la era de la globalización, México, Anthropos/UAM.

KANT, Immanuel, 1999, Hacia la paz perpetua. Un esbozo filosófico, Madrid, Biblioteca Nueva.

LINARES, Jorge Enrique, 2008, Ética y mundo tecnológico, México, FCE-UNAM.

Organización Mundial de la Salud, 2010, Informe sobre la salud en el mundo. La financiación de los sistemas de salud: el camino hacia la cobertura universal, OMS, disponible en: https://wwew.who.int/whr/2010/es/.

Pogge, Thomas, 2013, Hacer justicia a la humanidad, México, FGE-UNAM.

RAWLS, John, 2005, El derecho de gentes, Barcelona, Paidós. 